\title{
argestre
}

\section{Evolução da literacia digital e sua importância para os trabalhadores da saúde na era da inteligência digital}

\author{
EVOLUTION OF DIGITAL LITERACY AND ITS IMPORTANCE FOR HEALTH WORKERS IN \\ DIGITAL INTELLIGENCE AGE
}

Gilberto Vieira Branco', Paulo Roberto de Lima Lopes2, Ivan Torres Pisa3

\author{
1 Mestrando no programa de pós-graduação em \\ Gestão e Informática em Saúde. Universidade Federal \\ de São Paulo. \\ ORCID: https://orcid.org/0000-0002-2206-8773 \\ Email: gvbranco@unifesp.br \\ 2 Doutor em Ciências. Rede Nacional de Ensino e \\ Pesquisa - RNP. \\ ORCID: https://orcid.org/0000-0002-5730-1985 \\ Email: paulo.lopes@rnp.br \\ 3 Livre-docente, Departamento de Informática em \\ Saúde. Universidade Federal de São Paulo. \\ ORCID: https://orcid.org/0000-0002-5106-3904 \\ Email: ivanpisa@gmail.com
}

Correspondência: Departamento de Informática em Saúde EPM UNIFESP - Rua Botucatu 862 - Vila Clementino - 04023-062 - São Paulo SP.

Copyright: Esta obra está licenciada com uma Licença Creative Commons Atribuição-NãoComercial 4.0 Internacional.

Conflito de interesses: os autores declaram que não há conflito de interesses.

\section{Como citar este artigo}

Branco GV; Lopes PR de L; Pisa IT. Evolução da literacia digital e sua importância para os trabalhadores da área da saúde na era da inteligência digital. Revista de Saúde Digital e Tecnologias Educacionais. [online], volume 5, n. 3. Editor responsável: Luiz Roberto de Oliveira. Fortaleza, dezembro de 2020, p. 01-15. Disponível em: http://periodicos.ufc.br/resdite/index. Acesso em "dia/mês/ano".
Data de recebimento do artigo: $23 / 05 / 2020$

Data de aprovação do artigo: 19/08/2020

Data de publicação: 31/12/2020

\section{Resumo}

Introdução: Este estudo tem como objetivo identificar as aplicações do conceito de literacia digital na área de saúde digital e identificar instrumentos para medir o nível de literacia digital dos trabalhadores da saúde. Métodos: Trata-se de revisão integrativa de literatura com uso dos descritores "digital literacy" e "health" aplicados nas bases indexadas ERIC, Periódicos CAPES e PubMed. Foram localizados 121 artigos cuja seleção de 16 artigos possibilitou identificação de três frameworks internacionais. Resultados: Existem diferentes abordagens possíveis para o desenvolvimento da literacia digital como a prática baseada em evidências, desenvolvimento baseado em projetos, uso de redes sociais, elaboração e aplicação de frameworks, e aplicação de instrumentos de avaliação. Identificou-se que o framework de inteligência digital foi desenvolvido com base na compilação de outros 25 frameworks sobre literacia digital e que sua aplicação possibilita a avaliação de 24 competências em três níveis: cidadania digital, criatividade digital e competitividade digital. Conclusão: Existem modelos internacionais de avaliação de literacia digital e estudos que comprovam sua importância. Há uma lacuna para o desenvolvimento de um instrumento específico de autoavaliação de literacia digital com foco em 
trabalhadores da saúde no Brasil com base no modelo de inteligência digital.

Palavras-chave: literacia digital, saúde digital, inteligência digital.

\begin{abstract}
Introduction: This study aims to identify the applications of the concept of digital literacy in the area of digital health and identify instruments to measure the level of digital literacy of health workers. Methods: This is an integrative literature review using the descriptors "digital literacy" and "health" applied in ERIC, Periódicos CAPES and PubMed indexed databases; 121 papers were found whose 16 papers selection enabled the identification of three international frameworks. Results: There are different possible approaches to the development of
\end{abstract}

digital literacy such as evidence-based practice, project-based development, use of social networks, development and application of frameworks, and application of assessment tools. It was identified that the digital intelligence framework was developed based on the compilation of 25 other frameworks on digital literacy and that its application allows the evaluation of 24 competencies at three levels: digital citizenship, digital creativity and digital competitiveness. Conclusion: There are international models of digital literacy assessment and studies that prove its importance. There is a gap for the development of a specific digital literacy self-assessment instrument focused on health workers in Brazil based on the digital intelligence model.

Keywords: digital literacy, digital health, digital intelligence.

\section{Introdução}

Em 2017 o Ministério da Saúde apresentou sua estratégia para e-Saúde no Brasil ${ }^{1}$ em alinhamento com as orientações da Organização Mundial de Saúde (OMS). No documento, foram apresentados os quatro pilares da estratégia, caracterizando as áreas de governança e recursos organizacionais, padrões e interoperabilidade, infraestrutura e recursos humanos $(\mathrm{RH})$. Nesta última área, foram definidos vários objetivos, como a ampliação do acesso à atenção básica de saúde por meio do uso das tecnologias digitais da informação e das comunicações (TDIC), a qualificação das equipes de saúde com a promoção de educação permanente por meio de instrumentos de educação a distância (EaD), definição de perfil profissional de e-saúde e de uma matriz de competências com ênfase na valorização do profissional de saúde e conseguinte melhor atendimento para o paciente. Outra ação proposta pelo Ministério da Saúde foi a definição do público-alvo da estrutura de e-saúde no Brasil, descrita em dez categorias: 1. Pacientes, indivíduos e cidadãos; 2. Profissionais de saúde; 3. Gestores de saúde; 4. Prestadores de serviços de saúde; 5. Instituições de ensino e pesquisa; 6; Operadoras de planos de saúde; 7. Profissionais de informática em saúde; 8. Empresas fornecedoras de sistemas de informação em saúde; 9. Autoridades; 10. Outras entidades públicas e privadas. Destacamos as categorias de profissionais de saúde, gestores de saúde e profissionais de informática em saúde deste público-alvo para formar o escopo deste estudo, aqui denominados como trabalhadores da saúde. 
Este estudo tem como objetivo identificar as aplicações da literacia digital na área de saúde digital e identificar os instrumentos que possibilitem medir o nível de literacia digital dos trabalhadores da saúde. Como resultado iremos descrever a evolução do conceito de literacia digital, sua importância para os trabalhadores da saúde e quais são os conhecimentos, habilidades, valores e atitudes que compõe as competências ${ }^{2}$ que este grupo de profissionais precisa ter para favorecer sua atuação efetiva na área de saúde digital. Dessa forma será possível apresentar o conceito de literacia ${ }^{3}$ que está definida como a capacidade em ler, escrever e compreender de forma efetiva, e o conceito de literacia digital $^{3}$ que foi originalmente descrito como a capacidade para compreender, avaliar e integrar informações em diferentes formatos por meio do computador.

Foi importante perceber que os conceitos ganharam novos significados, não apenas pela existência de diferentes contextos, mas pela evolução das tecnologias. Em 2003, Hull et al. apresentaram, além do conceito de literacia, a existência de múltiplas literacias, entre elas a literacia em saúde ${ }^{4}$ e a literacia digital ${ }^{5}$. Este estudo não tem como objetivo tratar a literacia em saúde uma vez que este conceito tem ênfase na população, mas objetiva descrever a evolução do conceito de literacia digital ao longo dos anos, sua importância para os trabalhadores da saúde, bem como novas abordagens sobre o tema.

\section{Métodos}

Este estudo baseou-se numa revisão integrativa de literatura e considerou as seis etapas propostas por Souza, Silva e Carvalho6: elaboração da pergunta norteadora, busca na literatura, coleta de dados, análise crítica dos estudos incluídos, discussão dos resultados e apresentação da revisão integrativa. Para descrever as relações existentes entre literacia digital e a área de saúde foram elaboras as seguintes questões:

1. Qual a necessidade em compreender o grau de competência relacionado aos recursos das TDIC para um trabalhador da saúde?

2. Existem instrumentos que possibilitem ao trabalhador da saúde fazer uma autoavaliação de seus conhecimentos, habilidades e atitudes na área das TDIC?

A busca na literatura teve como parâmetro a pesquisa de artigos científicos nas seguintes bases indexadas: Education Resources Information Center (ERIC), Portal de Periódicos CAPES e PubMed. 0 critério de busca foi executado da mesma forma nas três bases com a aplicação dos descritores "digital literacy" $e$ "health" uma vez que o objetivo era identificar artigos que tratassem da literacia digital relacionada a área de saúde. Como 
critérios de inclusão consideramos artigos que tivessem relação com a área de literacia digital, cujo texto estivesse em inglês, português ou espanhol, elaborados em um período máximo de 7 anos e disponíveis na íntegra para leitura. A Figura 1 apresenta o fluxo da seleção e os motivos de exclusão dos artigos.

Foram obtidos 27 artigos na base ERIC e 94 artigos no PubMed. Dos 121 artigos encontrados, foram mantidos 9 dos 27 obtidos na base ERIC e 7 dos 94 oriundos do PubMed. Além disso foram realizadas buscas na base Periódicos Capes para ampliar os resultados e obter acesso a textos completos. Como forma de verificação e aprofundamento de alguns artigos foram utilizados dois livros e dois relatórios que tinham relação com o tema literacia digital. Os livros sobre literacia digital ${ }^{7-8}$ e o relatório com o framework para a área de enfermagem ${ }^{9}$ foram obtidos a partir da análise das referências dos artigos selecionados. 0 relatório sobre inteligência digital ${ }^{10}$ foi obtido após busca direta sobre modelos de literacia digital no Google.

\section{Resultados}

Em uma primeira análise foi possível identificar o aumento de interesse sobre a importância em desenvolver a literacia digital na área de saúde e isso ficou evidenciado pelo aumento no número de publicações relacionadas ao assunto nos últimos cinco anos conforme apresentado na Figura 2.

Os resultados serão apresentados em ordem cronológica a partir de 2012 quando Ellis, Bliuc e Goodyear avaliaram processos de investigação de estudantes de farmácia e apresentaram o termo "nativo digital" para representar os jovens estudantes que poderiam aprender a usar recursos digitais de forma mais rápida que pessoas mais velhas em razão do seu alto grau de envolvimento com tecnologia e que este processo teria impacto na forma como as universidades deveriam ensinar seus conteúdos ${ }^{11}$. Contudo, a condição de nativo digital não fez com que o processo de investigação, ou descoberta do conhecimento, fosse diferente quando relacionado ao fator idade. Evidenciou-se fatores, como o contexto em que o estudante estava inserido e a forma como foram realizadas pesquisas, tanto por contato pessoal como em formato digital. A percepção do processo demonstrou que o uso de tecnologias facilitou a busca de informações, mas não foi o fator determinante para o resultado dos processos de investigação realizados na área de farmácia.

Em 2014 o termo "nativo digital" recebeu a definição de "naturalmente competente"8 ao lidar com TDICs e foi acompanhado de um novo termo "imigrante digital", sendo este o indivíduo que busca aprender e acompanhar o uso de tecnologia, mesmo com as 
dificuldades que este aprendizado demandava. Estas definições foram a base para Stocchetti apresentar o termo competência digital e estabelecer três níveis de competência: domínio técnico e metodológico para obter informações, uso de criticidade e criatividade para acessar informações, e cultura de informação, o que inclui as considerações éticas e a função da mídia.

Um outro termo encontrado foi "era digital" $8 ; 12$ e uma definição atualizada de literacia digital surgiu em 2015 no estudo de Kaur et al. ${ }^{12}$ que identificou as seguintes habilidades para um indivíduo ser considerado literato digital: habilidades técnicas para usar efetivamente recursos tecnológicos, construir conhecimento ao navegar pela web, decifrar interfaces de usuários, realizar pesquisas em bases de dados, criar e distribuir conteúdo digital, conversar em salas de bate-papo e se comunicar em redes sociais. Outro fator importante encontrado neste estudo foram os aspectos de conhecimentos observados em indivíduos com literacia digital: ética, comprometimento pessoal, clima positivo e uso de ferramentas digitais.

A falta de habilidades de literacia digital em um ambiente de trabalho foi apresentada por Hill ${ }^{13}$, em 2016, como um limitador para a conquista de novas oportunidades de empregos, e até mesmo um fator que pode levar à exclusão social e à falta de equidade nas relações de trabalho. Hill ${ }^{13}$ também demonstrou a importância da integração do conceito de literacia digital com as disciplinas em um ambiente escolar. Em outro estudo, Tzoc e Ubbes ${ }^{14}$ apresentam a importância da divulgação e fortalecimento de ações que possibilitassem a ampliação da literacia digital da comunidade escolar em instituições de ensino e realizaram um estudo a partir da disciplina de educação em saúde. Em 2017 a instituição foco do estudo fomentou o aprendizado baseado em projetos para incentivar a democratização das habilidades de literacia digital. Para isso desenvolveram o Digital Literacy Partnership (DLP) que tinha como missão envolver bibliotecários, alunos e professores na elaboração de projetos educacionais e culminou na elaboração de uma página web que possibilitou o compartilhamento e acompanhamento dos projetos desenvolvidos em diferentes disciplinas.

Em 2018 Russel et al. ${ }^{15}$ descreveram os processos de educação na área de enfermagem na Austrália e evidenciaram a importância da prática baseada em evidências (PBE) na área de saúde como sendo uma das formas reconhecidas para a prática profissional de enfermagem e destacaram como benefício o apoio à tomada de decisões. Contudo, apontaram deficiências na aplicação prática da PBE por alguns profissionais de enfermagem que alegaram ser mais fácil a realização de pesquisas simples na web em 
decorrência de questões relacionadas à falta de tempo, falta de habilidade em pesquisas, dificuldade de compreensão de bases de dados eletrônicas e dificuldade em compreender artigos científicos. 0 estudo também apresentou a relação entre literacia digital e a literacia de informação de maneira que a primeira foi considerada uma multiliteracia e a segunda seria complementar por envolver capacidades sofisticadas, incluindo as habilidades cognitivas de alto nível: avaliação, interpretação e aplicação. Como forma de desenvolver as habilidades em PBE foi desenvolvida uma ferramenta instrucional com base na web para aproximar estudantes de enfermagem ao assunto.

Ainda na linha de educação em saúde, mas com uma estratégia desenvolvida com o apoio de redes sociais, Deaves et al. ${ }^{16}$ mostraram como o uso do Twitter pode ajudar na obtenção de conhecimento na área de saúde e no desenvolvimento de comportamentos positivos como networking, habilidades de comunicação e empregabilidade. Tais comportamentos reforçam a importância da literacia digital e o uso de relações em redes embasados pelas teorias de socioconstrutivismo, aprendizagem colaborativa e modelos de aprendizagem social, ainda que na área de saúde existam poucas evidências de eficácia das redes sociais com modelos associados a satisfação, conhecimento, habilidades e valores do estudante. 0 uso de redes sociais na área de saúde e a elaboração de cursos de pósgraduação aliados ao uso de plataformas digitais de acesso em massa também foram evidenciados por Mesko, Győrffy e Kóllar ${ }^{17}$ e Gálban e Vidal ${ }^{18}$ de forma a reforçar a estratégia de educação continuada para trabalhadores da saúde proposta pela OMS e apresentada na estratégia de e-saúde do Brasil ${ }^{1}$.

Com uma visão diferente sobre a importância da literacia digital na área de saúde, Sterponi et al. ${ }^{19}$ apresentaram um estudo que indicava as razões que poderiam dificultar o trabalho de médicos oncologistas ao usar recursos das TDICs em seus atendimentos. Nesse estudo foram apontados fatores como a ruptura na interação médico-paciente por diferentes motivos, entre eles a necessidade de atenção na digitação dos dados do paciente no Prontuário Eletrônico do Paciente (PEP), a demora para interagir com o paciente em razão do foco no computador, quebra de contato para acompanhar os dados apresentados na tela do computador, mudanças repentinas de assunto para atender as demandas apresentadas no sistema computacional e falta de espaço na mesa de trabalho em razão da presença do teclado. 0 contraponto dessa experiência foi o estudo promovido por King ${ }^{20}$ que afirmou que a literacia digital deveria ser o quarto pilar da educação em conjunto com leitura, escrita e matemática. De acordo com King ${ }^{20}$, a dificuldade em dominar a literacia digital ocorre em pessoas mais velhas, 55-65 anos, mas em geral as pessoas, novas e 
velhas, ainda têm dificuldade em compreender o que são informações e desinformações no cenário atual.

A busca por instrumentos que possibilitem uma autoavaliação resultou na identificação de três frameworks baseados na definição das competências e das áreas de desenvolvimento da literacia digital|-10;21. Um instrumento para investigação foi desenvolvido na Austrália para avaliar as atitudes das equipes de saúde em relação aos sistemas de informação, em um estudo conduzido por Kuek e Hakkennes ${ }^{5}$ em 2019, após identificar que existiam poucos instrumentos para avaliar a literacia digital de profissionais de saúde, e que os existentes eram muito longos, ou não tinham sido validados, ou eram específicos para um segmento na área de saúde. As autoras aplicaram o modelo de aceitação de tecnologia para avaliar as atitudes dos profissionais de saúde em um questionário com seis questões e fizeram a análise quantitativa das respostas.

No estudo australiano conduzido por McKinstry et al. ${ }^{21}$ foram apresentadas evidências da importância da literacia digital na área de saúde com foco em profissionais de Terapia Ocupacional (TO) que descreveram a criação de um modelo de literacia digital e uma ferramenta para mapear os conhecimentos, habilidades e valores dos profissionais de TO inspirados no livro de Belshaw 7 . O estudo apresenta um modelo criado com três níveis progressivos de desenvolvimento: básico, intermediário e avançado, e teve como base as seguintes premissas: criar compreensão, desenvolver a cultura, construir conexões, apropriar-se do espaço, usar capacidade total e pensamento transformador. Cada um dos temas foi relacionado com as práticas profissionais na área de TO e o desdobramento deste estudo foi a criação de uma plataforma sobre literacia digital para graduados na área de saúde e que está disponível em https://digitalliteraciesforhealthgraduates.com.au/.

Outros dois modelos foram identificados para o desenvolvimento da literacia digital e tinham em comum a abordagem baseada em competências ${ }^{9-10}$. No estudo de Hübner et al. ${ }^{9}$ foi apresentada uma recomendação de framework internacional para o desenvolvimento das competências digitais de profissionais de enfermagem que identificou 24 áreas de competências em informática em saúde e os cinco principais papéis de enfermeiros, sendo um com relação direta aos recursos das TDICs e foi classificado como gerenciamento de tecnologia da informação (TI). O outro framework foi desenvolvido pelo DQ Institute ${ }^{10}$ de Cingapura e tem como proposta o desenvolvimento do conceito de inteligência digital como sendo a evolução dos conceitos de quociente de inteligência e inteligência emocional. Em seu relatório foi apresentada uma matriz de competências elaborada a partir da análise de 25 frameworks de literacia digital existentes e apresentada com três níveis de 
conhecimentos: cidadania digital, criatividade digital e competitividade digital, organizados em oito áreas de desenvolvimento: identidade digital, uso digital, proteção digital, segurança digital, inteligência emocional digital, comunicação digital, literacia digital e direito digital. 0 framework apresenta um total de 24 competências a serem desenvolvidas nos indivíduos.

O desenvolvimento da literacia digital apoiada pela análise de competências ${ }^{14-16 ; 21}$ sugeriu o empoderamento dos trabalhadores da saúde que foi abordado por Mesko e Győrffy ${ }^{22}$ em 2019 no estudo que atestou que este trabalhador do século 21 deveria possuir habilidades como saúde digital, curiosidade, trabalho em equipe, aprendizagem contínua, criatividade, parceria com pacientes, empatia e literacia digital. 0 estudo propôs ainda os possíveis benefícios e as eventuais barreiras para que este empoderamento se tornasse realidade $22-23$. Algumas das barreiras apresentadas foram a falta de conhecimento sobre tecnologias de saúde digital, falta de equipe de apoio treinada, problemas com privacidade e segurança de dados do paciente e uma cultura de trabalho que rejeita inovações.

Tais problemas se aproximaram dos encontrados no estudo de Terry et al. ${ }^{24}$ no Reino Unido ao avaliarem a evolução de estudantes de enfermagem e obstetrícia a partir de um programa de treinamento em pares cujo objetivo era desenvolver a literacia digital. Naquele estudo foi possível identificar aumento da confiança dos estudantes e de suas habilidades como facilitadores, bem como benefícios percebidos pelos professores que incluíram especificamente um aumento do entendimento sobre experiências de aprendizagem, desenvolvimento de habilidades de ensino e autoconsciência.

\section{Discussão}

A análise dos artigos reforçou a importância em se compreender o grau de

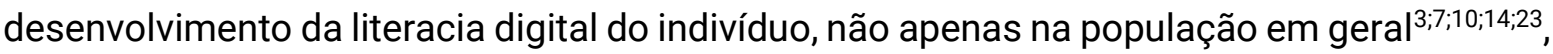
mas principalmente em trabalhadores da saúde desde sua formação inicial, tanto na modalidade de cursos técnicos como na graduação 9;21;24-25. Diferentes estratégias foram apresentadas para ampliar as habilidades na área de literacia digital, entre elas foram identificadas o uso de redes sociais, plataformas digitais de EaD, aprendizagem baseada em projetos e cursos formais de pós-graduação. Tais recursos foram apresentados como estratégias de educação continuada no Reino Unido, Austrália, Estados Unidos e no Brasil 1;; 12;14-15;17;24 em alinhamento com as diretrizes da OMS.

Foi possível abranger a evolução da literacia digital e sua relação direta com a oferta e uso de recursos das TDIC como facilidade de acesso à internet, o surgimento das redes sociais, a evolução das plataformas de colaboração, ensino virtual, telefones e smartphones, 
computação em nuvem etc. Na Figura 3 é possível observar a evolução do conceito de literacia digital ao longo dos últimos anos e tem como objetivo apresentar os elementos que foram adicionados à definição original de literacia digital feita em $1997^{3}$ e que estão relacionados com os aspectos do desenvolvimento de competências digitais ${ }^{7 ; 10}$ e das habilidades cognitivas ${ }^{21}$.

O processo de revisão possibilitou que fossem identificados estudos que apresentam instrumentos de avaliação baseados em métodos quantitativos com a aplicação de pesquisas online para identificar hábitos e habilidades ${ }^{17}$ e questionários sobre atitudes em relação à aceitação de tecnologia ${ }^{5}$. Também foram identificados instrumentos com a aplicação de métodos qualitativos com o objetivo de compreender as dificuldades relacionadas à falta de literacia digital ${ }^{23-25}$.

O modelo proposto por McKinstry et al. ${ }^{21}$ apresentou uma relação positiva e significativa ao buscar o desenvolvimento de competências a partir da tese de Belshaw ${ }^{7}$, oriunda da área de educação. Dessa forma foi possível compreender que a aplicação do conceito de desenvolvimento de competências composta por conhecimentos, habilidades, atitudes e valores, proposto pela Organização para a Cooperação e Desenvolvimento Econômico (OCDE), e utilizado nos três frameworks encontrados, indicam uma forma de se avaliar o desenvolvimento individual com base em métodos claros e bem definidos.

Ainda em relação ao uso de competências para se avaliar o grau de literacia digital, a análise do relatório do DQ Institute ${ }^{10}$ de Cingapura revelou que seu framework de inteligência digital foi construído a partir da análise de 25 frameworks de literacia digital, entre eles o da Microsoft ${ }^{5}$, Mozilla $^{7}$ e OCDE $^{9}$, o que mostra a robustez do modelo proposto e incentiva sua aceitação e aplicação tanto na área de educação como na área de saúde para a avaliação e desenvolvimento dos trabalhadores. Outro ponto importante do modelo de inteligência digital foi a definição de literacia digital apresentada pelo DQ Institute ${ }^{10}$ : "a habilidade em encontrar, ler, avaliar, sintetizar, criar, adaptar, e compartilhar informação, mídia e tecnologia".

Fica evidente a semelhança encontrada no modelo australiano ${ }^{21}$ e no framework do DQ Institute ${ }^{10}$ em segmentar por três níveis de conhecimento as áreas de desenvolvimento. A diferença entre os níveis propostos está na forma e na orientação. 0 modelo australiano classifica o nível de capacidade em básico, médio e avançado, e o modelo de Cingapura classifica o desenvolvimento com base no conjunto de competências propostas e apresenta os níveis de cidadania digital, criatividade digital e competitividade digital. Pode-se inferir que privilegiar a nomeação dos níveis em detrimento da simples gradação pode gerar maior 
interesse nos trabalhadores da saúde e, como consequência, espera-se uma aceitação do modelo de inteligência digital.

\section{Conclusão}

O termo literacia digital tem uma relação muito estreita com a área de educação e a forma como foram apresentadas as diversas definições sobre o tema foram importantes para se perceber a evolução do conceito, sua relevância para a área de saúde digital e a necessidade em se desenvolver ações de capacitação baseadas no modelo de competências relacionadas à literacia digital dos trabalhadores da saúde.

Esta revisão integrativa de literatura possibilitou uma compreensão da importância em se desenvolver a literacia digital dos trabalhadores da saúde para favorecer melhores práticas na área de saúde digital. Foi identificado que existem três frameworks propostos em nível internacional para o desenvolvimento da literacia digital, mas com focos diferentes. 0 modelo australiano ${ }^{21}$ tem foco no desenvolvimento de graduados em saúde, enquanto $o$ modelo alemão ${ }^{9}$ foi direcionado para o desenvolvimento dos profissionais de enfermagem e o modelo de Cingapura ${ }^{10}$ foi elaborado para a área de educação com a proposta de se avaliar a literacia digital de forma ampla e relacionada ao conceito de inteligência digital.

Foi possível concluir que existe uma lacuna para o desenvolvimento de um novo instrumento de investigação que possibilite aos trabalhadores da saúde no Brasil de realizar processos de autoavaliação abarcados pelo conceito de inteligência digital. Entende-se que uma ferramenta de autoavaliação pode auxiliar o processo de autoconhecimento, incentivar processos de educação continuada de forma estruturada de acordo com as necessidades de cada perfil profissional e usado como modelo de tomada de decisão para a realização de investimentos em treinamento e desenvolvimento dos trabalhadores da saúde de forma individual e coletiva.

\section{Referências}

1. Brasil. Estratégia de e-saúde para o Brasil. Brasília: Ministério da Saúde, Comitê Gestor da Estratégia e-Saúde; 2017.

2. OECD. OECD Future of Education and Skills 2030. [Online].; 2018 [cited 2020 may 17. URL: https://www.oecd.org/education/2030project/contact/E2030_Position_Paper_(05.04.2018).pdf. 3. Hull GA, Mikulecky L, St. Clair R, Kerka S. Multiple Literacies. A Compilation for Adult Educators. 2003. 
4. Quaglio G, Sørensen K, PR, Bertinato L, Brand H, TK, et al. Accelerating the Health Literacy Agenda in Europe. 2016; 32: p. 1074-1080.

5. Kuek A, Hakkennes S. Healthcare staff digital literacy levels and their attitudes towards information systems. Health Informatics Journal. 2019.

6. Souza MTd, Silva MDd, Carvalho Rd. Revisão integrativa: o que é e como fazer. Einstein. 2010; 8(1).

7. Belshaw D. The Essential Elements of Digital Literacies; 2014.

8. Stocchetti M. Media and Education in the Digital Age: Concepts, Assessments, Subversions Berlin: Peter Lang; 2014.

9. Hübner U, Shaw T, Thye J, Egbert N, Marin HdF, Chang P, et al. Technology Informatics Guiding Education Reform - TIGER - An International Recommendation Framework of Core Competencies in Health Informatics for Nurses. Methods Inf Med. 2018: p. e30-e42. 10. Institute DQ. DQ Global Standards Report 2019 - Common Framework for Digital Literacy, Skills and Readiness. Cingapura: DQ Institute; 2019.

11. Ellis RA, Bliuc AM, Goodyear P. Student experiences of engaged enquiry in pharmacy education: digital natives or something else? High Educ. 2012: p. 609-626.

12. Kaur S, Sidhu GK, Fong II, Jamian IS. Supervisory and digital literacy practices in postgraduate supervision: a case study. In society iiafdoti, editor. Proceedings of the 12th international conference on cognition and exploratory learning in the digital age (celda 2015); 2015. P. 35-42.

13. Hill L. Digital Literacy Instruction for eHealth and Beyond. ORTESOL Journal. 2016. 14. Tzoc E, Ubbes VA. The Digital Literacy Partnership Website: Promoting Interdisciplinary Scholarship between Faculty, Students, and Librarians. New Review of Academic Librarianship. 2017.

15. Russell F, Rawson C, Freestone C, Currie M, Kelly B. Parallel Lines: A Mixed Methods Impact Analysis of Co-Curricular Digital Literacy Online Modules on Student Results in FirstYear Nursing. College \& Research Libraries. 2018 Nov: p. 948-971.

16. Deaves A, Grant E, Trainor K, Jarvis K. Students' Perceptions of the Educational Value of Twitter: A Mixed-Methods Investigation. Research in Learning Technology. 2019.

17. Mesko B, Gyorffy Z, Kollár J. Digital Literacy in the Medical Curriculum: A Course With Social Media Tools and Gamification. JMIR Medical Education. 2015: p. e6.

18. Galbán PA, Vidal NV. Digital Literacy Program for the Use of Social Media, Aimed at Health Professionals. MEDINFO 2019: Health and Wellbeing e-Networks for All. 2019. 19. Sterponi L, Zucchermaglio C, Alby F, Fatigante M. Endangered Literacies? Affordances of Paper-Based Literacy in Medical Practice and Its Persistence in the Transition to Digital Technology. Written Communication. 2017: p. 359-386.

20. King K. Education, Digital Literacy and Democracy: The Case of Britain's Proposed 'Exit' from the European Union (Brexit). Asia Pacific Education Review. 2019: p. 285-294.

21. McKinstry C, lacono T, Kenny A, Hannon J, Knight K. Applying a digital literacy framework and mapping tool to an occupational therapy curriculum. Aust Occup Ther $\mathrm{J}$. 2019: p. 1-8.

22. Mesko B, Győrffy Z. The Rise of the Empowered Physician in the Digital Health Era: Viewpoint. J Med Internet Res 2019;21(3):e12490. 2019: p. e12490.

23. Almond $\mathrm{H}$, Cummings $\mathrm{E}$, Turner $\mathrm{P}$. Empowerment, Digital Literacy and Shared Digital Health Records: The Value of 'nothing about me without me'. Digital Health: Changing the Way Healthcare is Conceptualised and Delivered. 2019.

24. Terry J, Davies A, Williams C, Tait S, Condon L. Improving the digital literacy competence of nursing and midwifery students: A qualitative study of the experiences of NICE student champions. Nurse Education in Practice. 2018: p. 192-198. 
25. Campbell CJ, McDowell DE. Computer Literacy of Nurses in a Community Hospital: Where Are We Today? J Contin Educ Nurs. 2011: p. 365-370. 


\section{Anexos}

Figura 1 - Fluxo de seleção dos artigos.

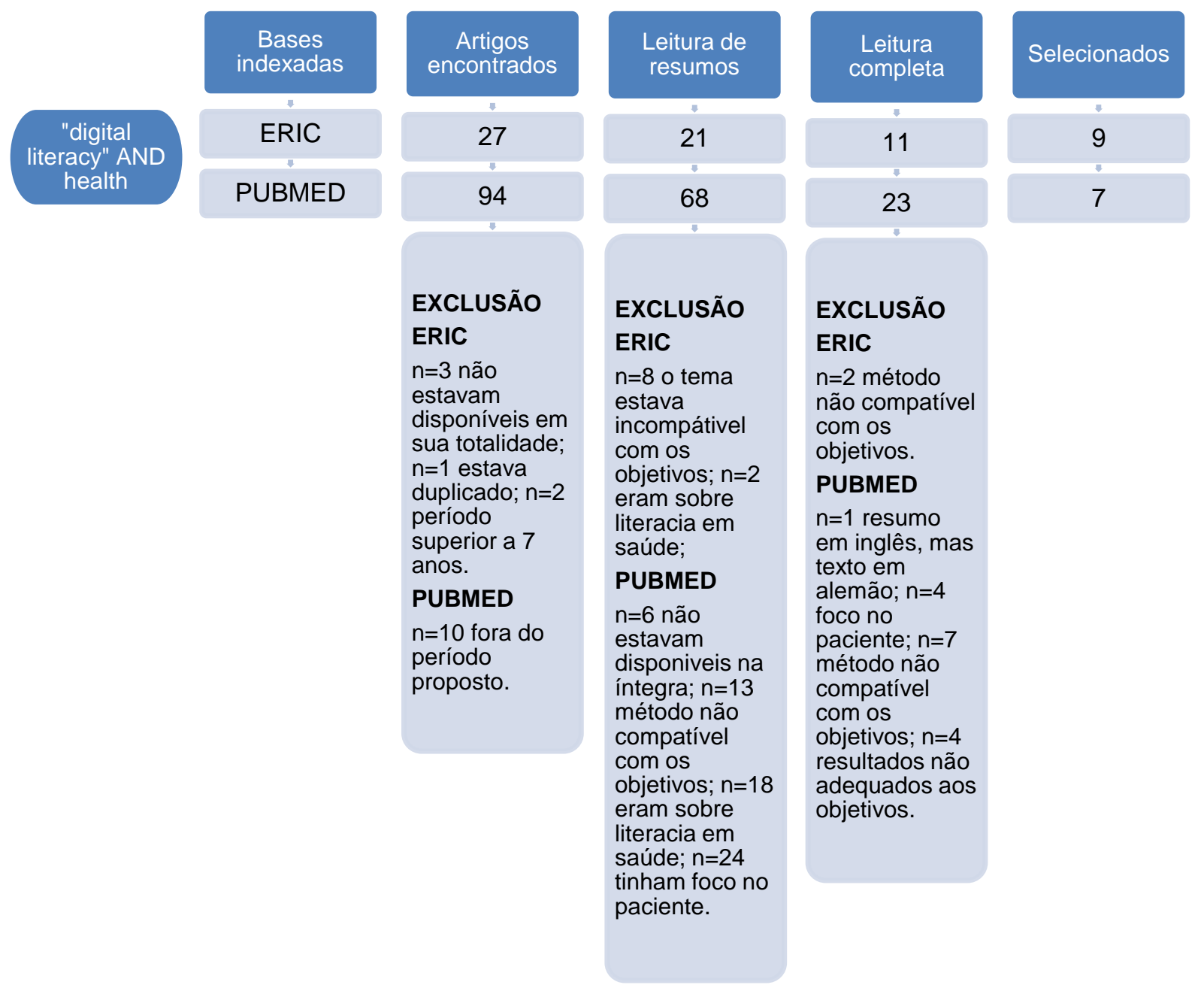

Fonte: os autores. 
Figura 2 - Quantidade de artigos sobre literacia digital.

Comparação da quantidade de artigos encontrados sobre literacia digital em duas bases indexadas

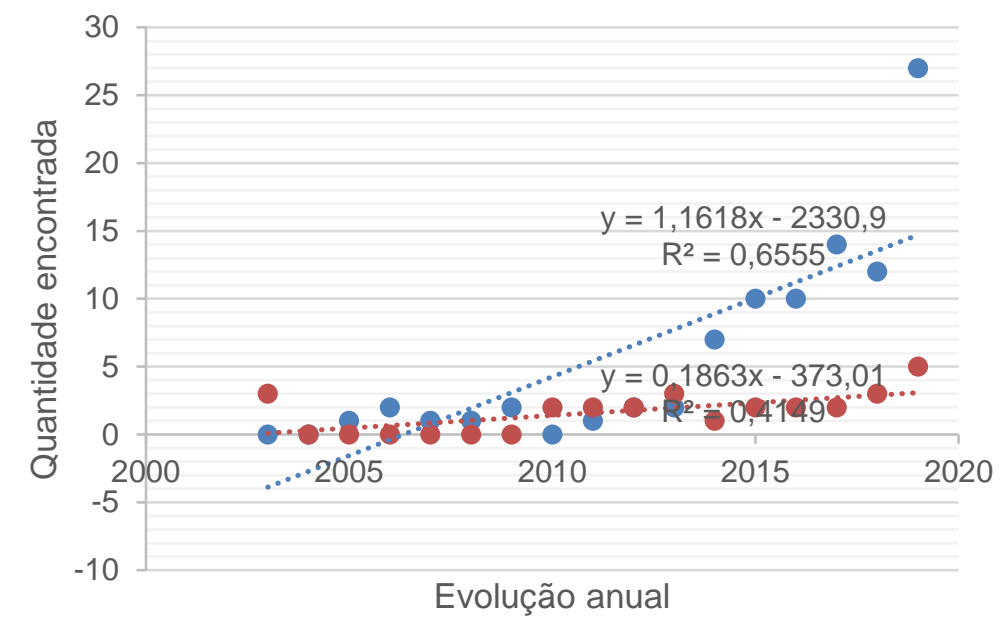

- Quantidade de Artigos no PUBMED

- Quantidade de Artigos no ERIC

- Linear (Quantidade de Artigos no PUBMED )

- Linear (Quantidade de Artigos no ERIC )

Fonte: os autores. 
Figura 3 - Evolução do conceito de literacia digital.

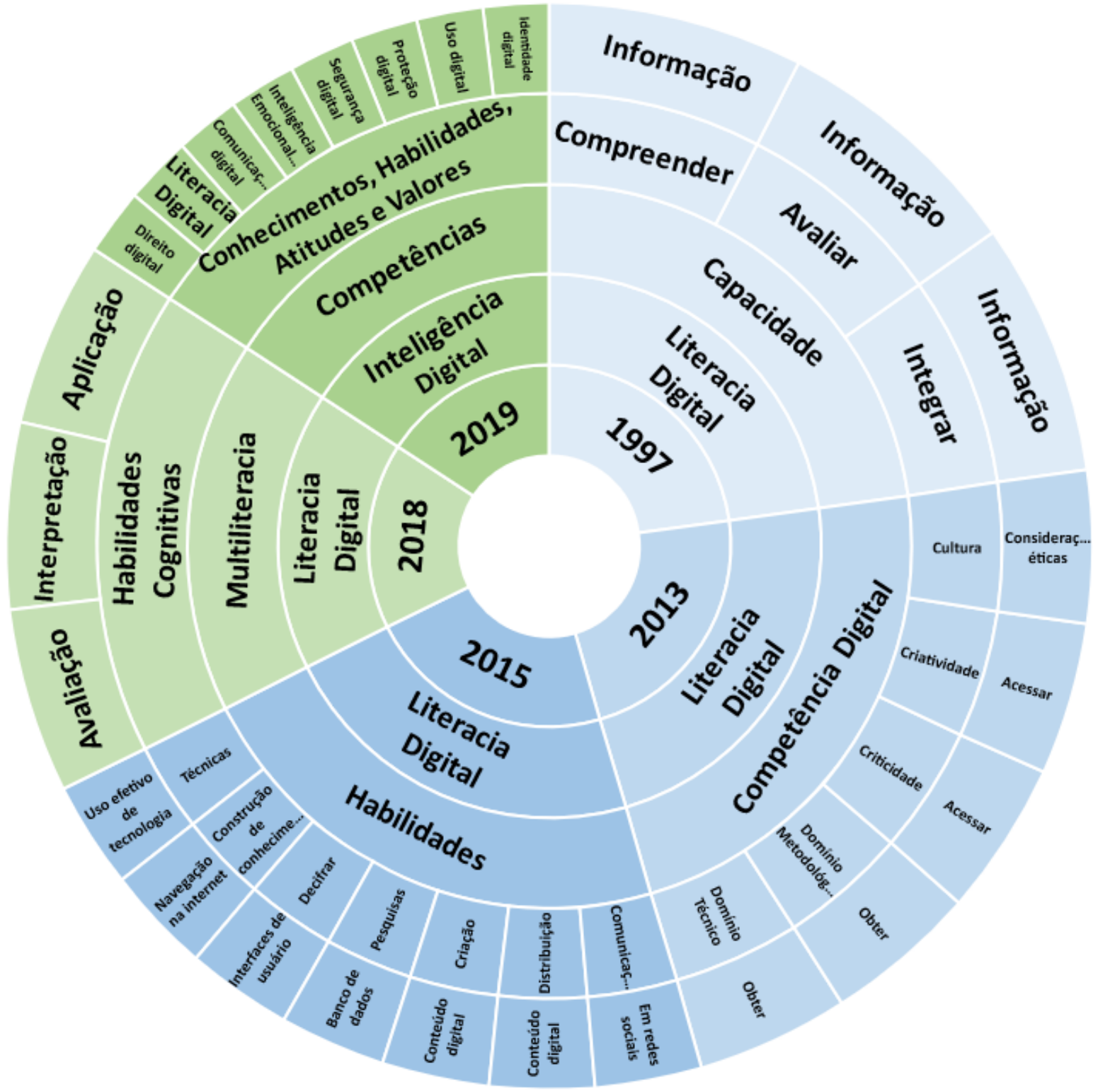

Fonte: os autores. 